\title{
Robust Pixel-wise Dehazing Algorithm Based on Advanced Haze-relevant Features
}

\author{
Guisik Kim \\ specialre@naver.com \\ Junseok Kwon \\ jskwon@cau.ac.kr
}

\author{
School of Computer Science and \\ Engineering \\ Chung-Ang University \\ Seoul, Korea
}

\begin{abstract}
The dehazing algorithm aims to remove haze from an image and has been widely used as a pre-processing step in several computer vision applications. The performance of the computer vision algorithm, however, is significantly affected by weather conditions. Hence, conventional algorithms do not work well when weather conditions vary severely over time. This paper proposes an effective haze removal algorithm based on a single image, which is robust to the varying weather conditions. Unlike conventional methods, which estimate "global" atmospheric light, we find the "local" atmospheric light by assuming that it is absorbed and emitted differently at each atmospheric particle. We divide the image into two components, namely, illumination and reflection, according to the retinex theory, and obtain the pixel-wise atmospheric light from the illumination. To estimate an accurate transmission, we initialize it by using a combination of haze-relevant features that have been proven experimentally to be highly correlated with haze. The aforementioned processes interact with each other in producing accurate dehazing results. We solve the dehazing problem via convex optimization and obtain the optimal solution. The experimental results demonstrate that the proposed algorithm outperforms state-of-the-art methods, using the benchmark dataset with regard to contrast, detail, and visibility. Quantitatively, our method produces better dehazing results than other conventional methods.
\end{abstract}

\section{Introduction}

In order for computer vision systems (e.g. object recognition, detection, and tracking systems) to perform as well as possible, the input image should be clear. An image captured outdoors, however, can be easily contaminated by several factors such as light scattering, absorbing, haze, smoke etc. The contaminated image has low contrast and faded color, which reduces overall visibility and degrades the computer vision algorithm. Hence, for the algorithms utilizing images captured outdoors, the haze removal process is a necessary pre-processing step.

Conventional haze removal algorithms [ $\square, \square, \square]$ typically utilize multiple information such as depth, 3D model, multiple images, and sensors. Recently, the algorithms utilizing just a single image have shown considerable success with dehazing problems $[\boldsymbol{\nabla}, \mathbb{\square}]$. Most single image-based algorithms [ $[\square, \mathbb{G}, \mathbb{\Xi}]$ ] handle the ill-posed dehazing problem with dark channel prior, while others $[\square]$ solve the problem by using multiple images generated from 


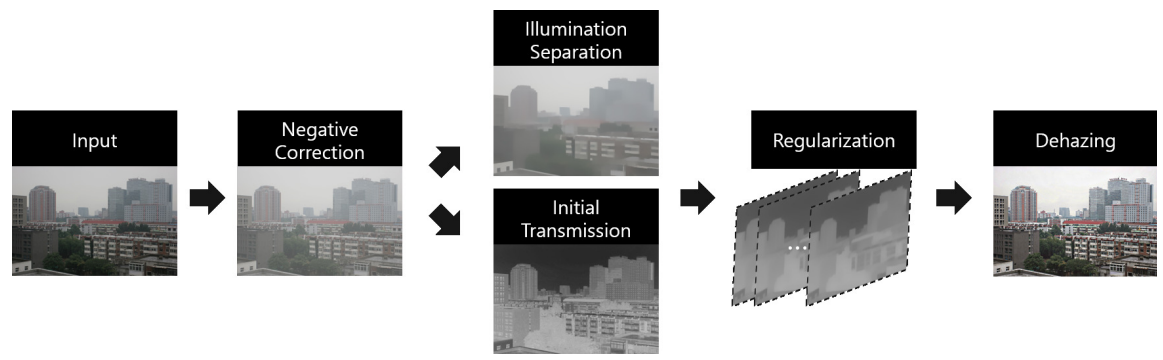

Figure 1: Overall procedure of the proposed algorithm.

a single image. Variant algorithms [ $[\mathbf{\square}, \mathbf{⿴}]$ improve the contrast and image field-of-view of without estimating atmospheric light and transmission. Dehazing algorithms typically assume that the input image is captured during daytime. Studies [ $[,, \mathbb{U}, \mathbb{\Xi}]$ mitigated this assumption and considered the dehazing problem during nighttime. A nighttime haze image includes multiple light sources, as well as atmospheric light; therefore, it is difficult to obtain an accurate haze removal image. The method [ $[$ ] $]$ overcomes the above problem by accurately describing additional light sources in the image. Recently deep convolutional neural network based methods $[\boldsymbol{Q}, \mathbf{E}]$ ] have emerged and have produced promising results. The aforementioned methods, however, have a common problem, which is caused by considering atmospheric light as global.

In this paper, we argue that the haze images under real-world environments can not be described by the "global" atmospheric light, and we propose a new dehazing algorithm. Given an input image, we first adjust brightness using a negative correction. In order to estimate pixel-wise atmospheric light from the adjusted image, we obtain the illumination from the image and use it to extract haze-relevant features. The accurate initial transmission is then estimated. After regularization, the final transmission is generated and we can obtain the haze removed image. Fig. 1 shows the overall procedure of our algorithm. Our proposed method makes four contributions; First, it utilizes the local atmospheric light during haze removal. To the best of our knowledge, there is no other algorithm that uses pixel-wise atmospheric light estimation. Second, it estimates the initial transmission by using hazerelevant features. With theses features, an accurate transmission is obtained. Third, we propose a dehazing algorithm, which can be used both during day and nighttime. Existing algorithms developed for addressing the daytime haze removal problem cannot be used in addressing the nighttime problem. The proposed method, however, can be used in both cases. Fourth, we demonstrate that our method outperforms state-of-the-art algorithms with regard to daytime haze removal. Additionally, our method is competitive with algorithms used for nighttime haze removal. By using the proposed method, we obtain the optimal solution to the dehazing problem via convex optimization and improve the contrast and details in haze removal images. The remainder of this paper is organized as follows: Section 2 introduces related work; Section 3 explains the proposed algorithm; in Section 4, the performance of the proposed method is evaluated based on the benchmark dataset and comparison is made to state-of-the-art methods; Section 5 concludes the paper with a summary of our study.

\section{Related Work}

To obtain good haze-free images, the methods in $[\square, \square, \square]$ used multiple images captured from different views to extract the scene depth information. The 3D geographic model was 
then constructed and used as an initial transmission during the dehazing process. He et al. [ㅁ] observed the statistical characteristics of haze-free images and proposed dark channel prior. They found that at least one of R, G, B channel had a low brightness value in 5,000 images, in which the sky region was manually removed. Hence, if some regions had high brightness values, the region could be considered a haze region. Tang et al. [ $\square]$ ] constructed a regression model, based on random forest, using several haze-relevant features. Tarel et al. []] proposed the "Median of Median Along Lines" algorithm, which preserved both edges and corners in the haze-free image by suppressing the halo effect during the veil estimation process. Schaul et al. [ $\square]$ used the infrared image to make a better, haze-free image. Tan et al. [四] removed haze from an image using the additional depth data. Meng et al. [ॄ] presented a boundary constraint and contextual regularization to accurately estimate the transmission. Yeh et al. [ $[\mathbf{⿴}]$ estimated the atmospheric light accurately by using pixel-based dark and bright channels. Kim et al. [ $[\mathbf{0}]$ employed a quad-tree method to estimate the atmospheric light; then, they estimated the transmission to improve contrast. Gao et al. [四] suppressed the halo effect with a maximum value filter and performed a negative correction to solve the over-saturation problem in the haze-free image. Recently Choi et al. [ [ $]$ ] provided a dehazing evaluation method and a real-world dataset.

To efficiently detect the haze, Ancuti et al. [四] proposed the "Semi-Inverse" method. Fattal et al. [ $[$ ] assumed that pixel values of haze regions are on the same line in the RGB space. Berman et al. [ $⿴ 囗 十$ ] proposed a non-local algorithm based on the observation that a haze-free image is represented by clusters containing hundreds of distinct colors. To find optimal solutions to the dehazing problem, He et al. [四] proposed a fast convex optimization algorithm by applying the discrete haar wavelet transform. Nishino et al. [四] presented a stochastic model based on a factorial Markov random field. This method estimated the scene albedo and depth and derived latent statistical structures in the image.

Owing to the fact that the algorithms described above assume a global atmospheric light they have difficulty describing white objects or other light sources in the image. Inaccurate transmission, along with the erroneous atmospheric light, causes saturation and overenhancement. In this paper, we propose a solution to the aforementioned problem, which consists of estimating the atmospheric light in a pixel-wise manner and by initializing the transmission using a new haze-relevant feature.

\section{Proposed Method}

The objective of dehazing problems is to improve the details and visibility of a haze image with accurate atmospheric light and transmission estimation. To achieve this goal, we use an algorithmic approach based on convex optimization [四]. We use the haze model proposed by Koschmieder et al. [四]. The haze image $I$ is generated by combining the haze-free image $J$ and the global atmospheric light $A$ with the weight $t$.

$$
I(\mathbf{X})=J(\mathbf{X}) t(\mathbf{X})+A(1-t(\mathbf{X})),
$$

where $t$ is the transmission, which is the amount of light transmitted from the camera. The value of $t$ is typically dependent on the distance from the camera. In (1), the first and second terms represent direct attenuation and air light, respectively. The estimation of $A$ and $t$ is described in Section 3.1 and Section 3.2, respectively. Convex optimization is explained in Section 3.3, which produces a haze-free image $J$. 


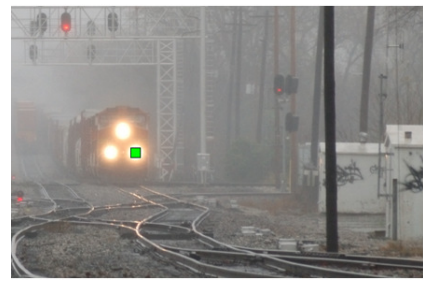

(a)

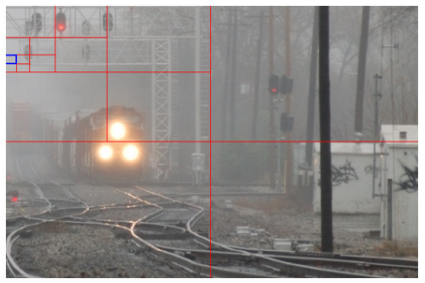

(b)

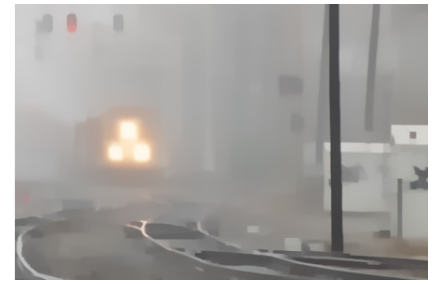

(c)

Figure 2: A atmospheric light estimation. (a) He's method [ष]]. (b) Quad-tree searching method [ㅁ] . (c) Proposed pixel-wise atmospheric light method.

As the first step of our dehazing algorithm, we apply the negative correction technique [ए] to the image to prevent it from becoming dark during the dehazing process. The negative correction of the two-dimensional coordinate $\mathbf{X}=(x, y)$ of the image is formulated by

$$
I(\mathbf{X})=1-F(\hat{I}(\mathbf{X}))
$$

where $\hat{I}(\mathbf{X})$ denotes the inverted value of the coordinate $(x, y)$, which is normalized to lie between 0 and 1. In (2), $F(\hat{I}(\mathbf{X}))$ indicates a linear transformation function, which calculates brightness correction in the inverted image. The result of $F(\hat{I}(\mathbf{X}))$ is inverted back into $I(\mathbf{X})$. With the use of negative correction, we can prevent the image from falsely having high brightness areas during the dehazing process. The negative correction result is used as the input for subsequent processing.

\subsection{Pixel-wise Atmospheric Light Estimation}

The estimation of atmospheric light $A$ is a challenging problem. Depending on the $A$ value, color can be distorted or saturated. Various estimating methods have been proposed to get an accurate $A$ value. He's method [ㅁ] , which estimates the highest value in the dark channel is commonly used; however, it is problematic when selecting a white object or a light source, as shown in Fig. 2 (a). The quad-tree searching method []], which divides the image and finds a point with a high average brightness, has been proposed as a method for solving the aforementioned problem; however, the area found by this method frequently converges into a white object, as shown in Fig. 2 (b).

Most existing dehazing algorithms estimate the atmospheric light as global and use it as one fixed value. In real-world environments, however, all the particles in the atmosphere absorb and emit light independently; therefore, each one of them acts as a light source. Based on this fact, we estimate the atmospheric light in a pixel-wise manner, assuming that the illumination of each pixel can be used as the atmospheric light. According to the retinex theory, the image $I(\mathbf{X})$ can be decomposed into illumination $L(\mathbf{X})$ and reflectance $R(\mathbf{X})$ for each channel.

$$
I^{c}(\mathbf{X})=L^{c}(\mathbf{X}) \times R^{c}(\mathbf{X}) \forall c=1,2,3,
$$

In (3), $L(\mathbf{X})^{c}$ can be obtained by the following cost function [四]:

$$
L^{c}(\mathbf{X})=\int\left(\left\|\nabla L^{c}(\mathbf{X})\right\|^{2}+\alpha\left\|L^{c}(\mathbf{X})-I^{c}(\mathbf{X})\right\|^{2}\right) d x,
$$

where $\nabla$ denotes the first-order differential operator, $\|\cdot\|$ returns the absolute value, and $\alpha$ is a weighting parameter. The first term in (4) imposes the smoothness constraint of the 


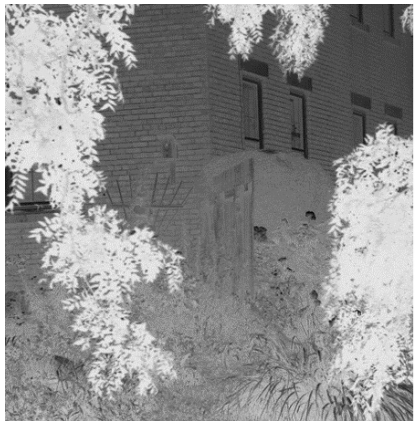

(a)

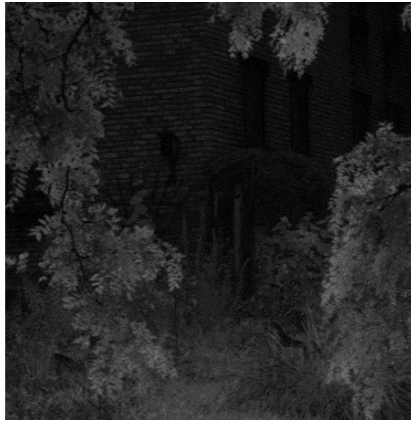

(b)

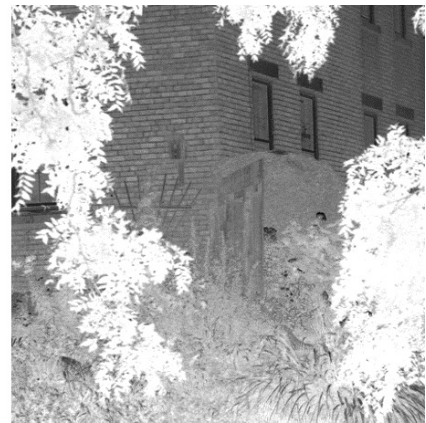

(c)

Figure 3: Estimation of the initial transmission. (a) Inversion Colour Attenuation. (b) White - Dark channel. (c) Proposed initial transmission.

illumination and the second term forces the illumination to have an intensity similar to $I(\mathbf{X})^{c}$. Then $L^{c}(\mathbf{X})$ in (4) is obtained through a gradient-descent algorithm [ $\left.\mathbb{} \mathbf{Q}\right]$ ]. Based on Shen's method $[\square]$, we apply this process to all the colour channels, $R, G$, and $B$. We make the illumination smoother over the pixels by performing relative total variation filtering (RTV) [四]. The estimated illumination is then used as a pixel-wise atmospheric light. Fig. 2(c) shows our atmospheric light results.

\subsection{Robust Initial Transmission Estimation}

The transmission $t$ plays a very important role in the dehazing algorithm along with the atmospheric light. An erroneous estimation of $t$ can result in a reconstructed image with numerous haze regions remaining. Therefore, it is necessary to estimate a more accurate initial transmission to obtain a better haze-free image. In this paper, we utilizes haze-relevant features, which are highly correlated with haze. We obtain the haze-relevant feature by using modified colour attenuation [曰] and dark channel [ $[\mathbf{\square}]$, in which colour attenuation can be expressed as follows:

$$
C(\mathbf{X})=I^{*}(\mathbf{X})-\frac{\max _{c \in r, g, b} I^{c}(\mathbf{X})-\min _{c \in r, g, b} I^{c}(\mathbf{X})}{\max _{c \in r, g, b} I^{c}(\mathbf{X})},
$$

where $I^{*}(\mathbf{X})$ denotes the maximum value of each channel per pixel.

The haze-free image typically has vivid colors. On the other hand, the haze image is faded from its original colors, resulting in saturation into high colors. Therefore, features based on the saturation information are appropriate for detecting haze. In (5), we assess whether a region in the image is saturated by using the second term, which has a small value when the region is highly saturated. Hence, colour attenuation $C(\mathbf{X})$ has a large value in haze regions. It has to be noted that the first term is a bias term.

To obtain a more robust haze-relevant feature, we also define the white channel and compute the difference between the white and dark channel:

$$
W D(\mathbf{X})=W(\mathbf{X})-D(\mathbf{X}),
$$

where $W(\mathbf{X})$ and $D(\mathbf{X})$ are the white channel and dark channel, respectively. In (6), $W D(\mathbf{X})$ is equal to the difference between the minimum and maximum values of a region. Hence 


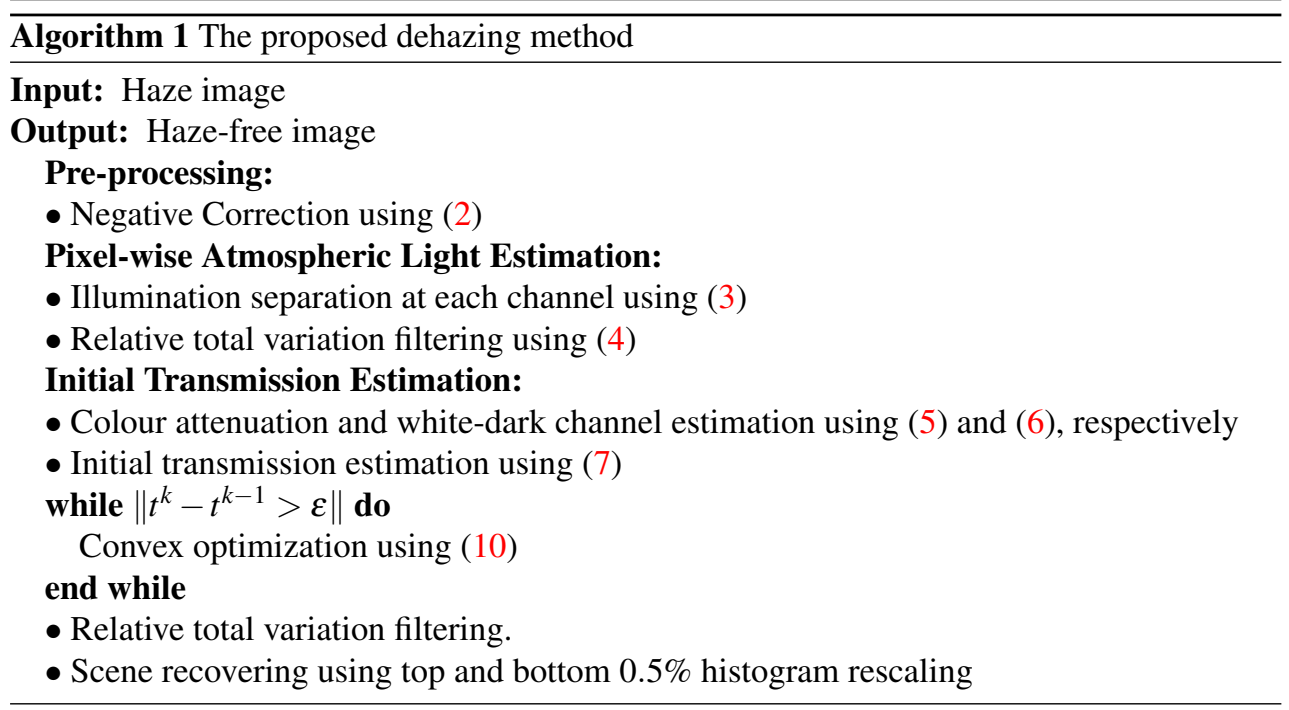

$W D(\mathbf{X})$ has a small value in the haze-contaminated regions. The proposed initial transmission is then defined as follows:

$$
t(\mathbf{X})=1-C(\mathbf{X})+W D(\mathbf{X}) .
$$

Please note that the initial transmission $t(\mathbf{X})$ is estimated in a pixel-wise manner and thus preserves details of the image, as shown in Fig.3, while the dark channel feature [四] is obtained in a block-wise manner.

\subsection{Convex Optimization}

The Contrast $(\mathbf{X})$ is modeled by

$$
\operatorname{Contrast}(\mathbf{X})=E\left[\left(J(\mathbf{X})-\mu_{J}\right)^{2}\right] \approx \frac{1}{t^{2}} E\left[\left(I(\mathbf{X})-\mu_{I}\right)^{2}\right],
$$

where $\mu_{J}$ and $\mu_{I}$ denote the mean of $J(\cdot)$ and $I(\cdot)$, respectively. In (8), we can make an approximation because $J(\mathbf{X}) \approx \frac{I(\mathbf{X})}{t}$. (8) shows that contrast is inversely proportional to transmission $t$. The haze image typically has low contrast values compared to the haze-free image. Based on this observation, we minimize $t$ to construct the haze-free image. Hence, the cost function is defined by

$$
\arg \min _{t} \lambda_{1}\|t\|_{2}^{2}+\lambda_{2}\|t\|_{T V}+\lambda_{3}\|Q\|_{2}^{2}
$$

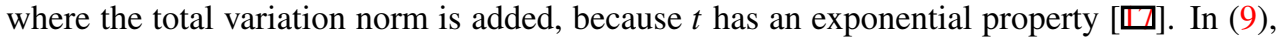
$Q=J t$ is also introduced, which makes the cost function a convex one.

Then, we transform the dehazing problem into a convex optimization problem. The convex optimization formula is as follows:

$$
\begin{aligned}
& \arg \min _{Q, t}\|I-(Q+A(1-t))\|_{2}^{2}+\lambda_{1}\|t\|_{2}^{2}+\lambda_{2}\|t\|_{T V}+\lambda_{3}\|Q\|_{2}^{2}, \\
& \text { s.t. } 0<t \leq 1,0<Q
\end{aligned}
$$




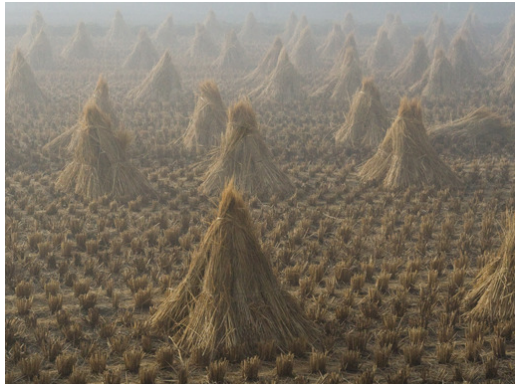

(a)

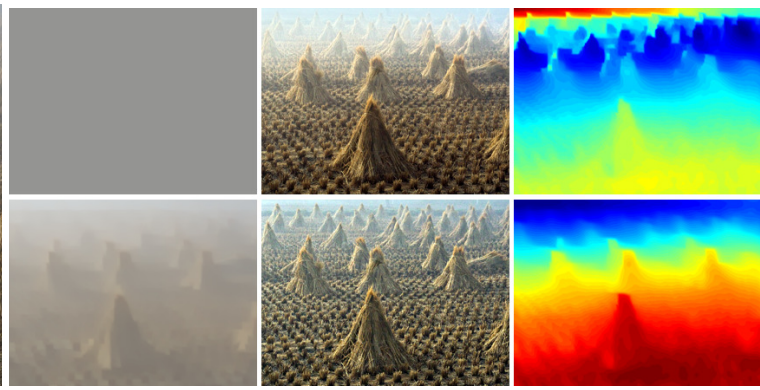

(b)

Figure 4: Performance of pixel-wise $A$. (a) Input image. (b) $1^{\text {st }}$ row: ATM [四] using global $A, 2^{\text {nd }}$ row: proposed method using pixel-wise $A, 1^{\text {st }}$ column: atmospheric light, $2^{\text {nd }}$ column: dehazing results, $3^{\text {rd }}$ column: transmission.

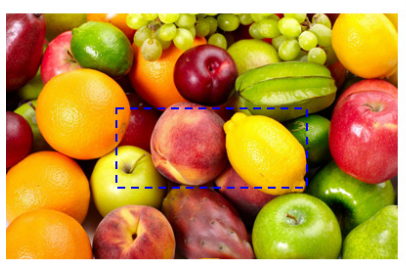

(a)

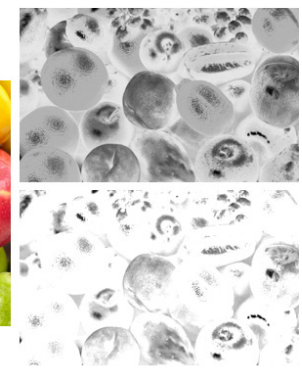

(b)
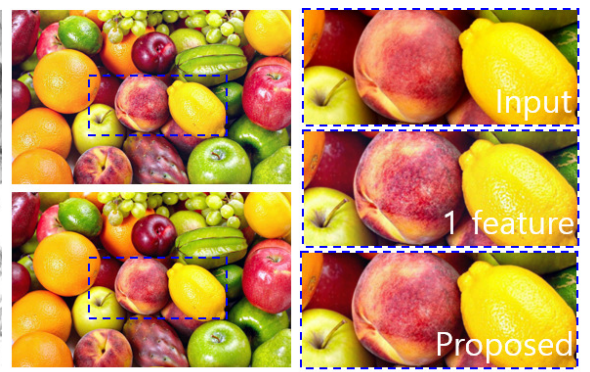

(c)

Figure 5: Performance of haze-relevant features. (a) Haze-free image. (b) Initial transmissions. (c) Dehazing results.

where the first term forces the reconstructed image $(Q+A(1-t))$ based on the haze model in (1) into being similar to the original image $I$. This proposed method adopts Split Bregman

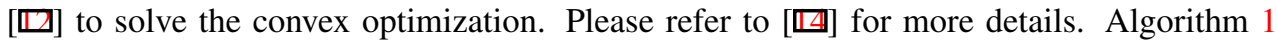
describes the overall procedure of the proposed method.

\section{Experiments}

Our algorithm was compared with the state-of-the-art methods: color-line [ $\mathrm{\theta}]$, haze-line [ $⿴$ ], and MSCNN [ $[\mathbb{G}]$. For the comparison, we used 35 test images provided by Fattal [ $\mathrm{\theta}]$. In the house image, the haze-line method set the atmospheric light to 1 and other methods set the light to ATM [四]. For the negative correction, with the linear transformation function in (2), our method takes $70 \%$ of the inverted intensity for each pixel.

\subsection{Component-wise Analysis for Proposed Method}

Our method consists of several components. It is worth determining which component contributes most to the success of the whole process. Hence, we conducted two experiments. Fig.4 shows the experimental results of $A$ using ATM [ $[$ 四] and proposed pixel-wise $A$. In the ATM, the transmission did not describe the depth accurately. Generally, it had a lower value than that of the haze-opaque region. When the proposed pixel-wise $A$ was used, however, the depth of the transmission was accurately estimated and the visibility was improved. 


\begin{tabular}{c|ccc|ccc|ccc|ccc}
\hline & \multicolumn{3}{|c|}{ MSCNN [四] } & \multicolumn{3}{c|}{ Color-line [日] } & \multicolumn{3}{c|}{ Haze-line [四] } & \multicolumn{3}{c}{ Proposed } \\
\hline & $e$ & $\sigma$ & $\bar{r}$ & $e$ & $\sigma$ & $\bar{r}$ & $e$ & $\sigma$ & $\bar{r}$ & $e$ & $\sigma$ & $\bar{r}$ \\
\hline House & 0.02 & 2.85 & 1.56 & 0.07 & 2.53 & 2.01 & 0.08 & 0.21 & 1.97 & 0.09 & 0.01 & 1.80 \\
Buildings & 0.06 & 0.89 & 1.14 & -0.08 & 7.12 & 2.93 & 0.00 & 10.5 & 1.78 & 0.03 & 0.34 & 2.39 \\
Ny12 & 0.06 & 0.01 & 1.13 & -0.16 & 0.43 & 1.82 & -0.08 & 1.39 & 1.80 & 0.01 & 0.15 & 1.87 \\
Urbino & -0.16 & 25.2 & 0.92 & 0.15 & 3.02 & 1.49 & 0.00 & 2.14 & 1.23 & 0.18 & 0.35 & 1.75
\end{tabular}

Table 1: Results for dehazing method. $e$ : newldy visible edge after dehazing, $\sigma$ : saturation pixel ratio, $r$ : quality of contrast restoration [ $\square]$.
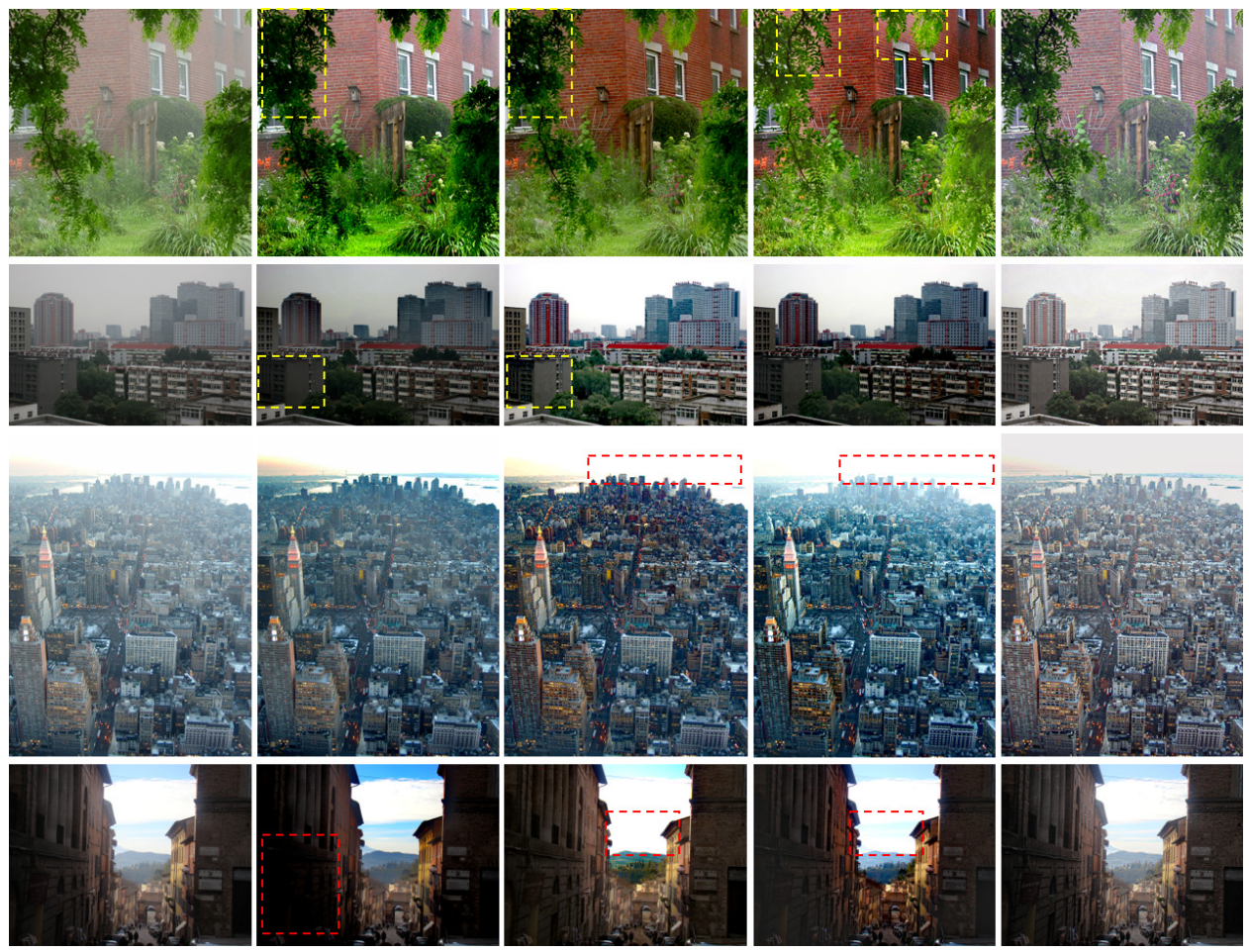

Figure 6: Day-time dehazing results. Left to Right, Input image, MSCNN [ㅈ] , Color-line [日], Haze-line [四] and Proposed method.

In Fig.5, we conducted an experiment with regard to haze-relevant features. With the proposed haze-relevant features, our method improved the initial transmission compared to the method in which only Colour Attenuation was used, as shown in Fig.5 (b). The proposed haze-relevant features also enhanced dehazing results, as demonstrated in Fig.5 (c). Combining haze-relevant features with white channel features resulted in less overenhancement of the haze-free area.

\subsection{Comparison with Other Methods}

We used the VLD [ [] ] as quantitative evaluation metrics, which quantified edge, saturation, and contrast. Table 1 demonstrates that the proposed method outperforms other state-of-theart methods; most images were significantly improved. In our method, saturation did not occur and the original pixel value was less than $1 \%$ such that $\sigma$ exceeded the $0 \sim 1$ range of 

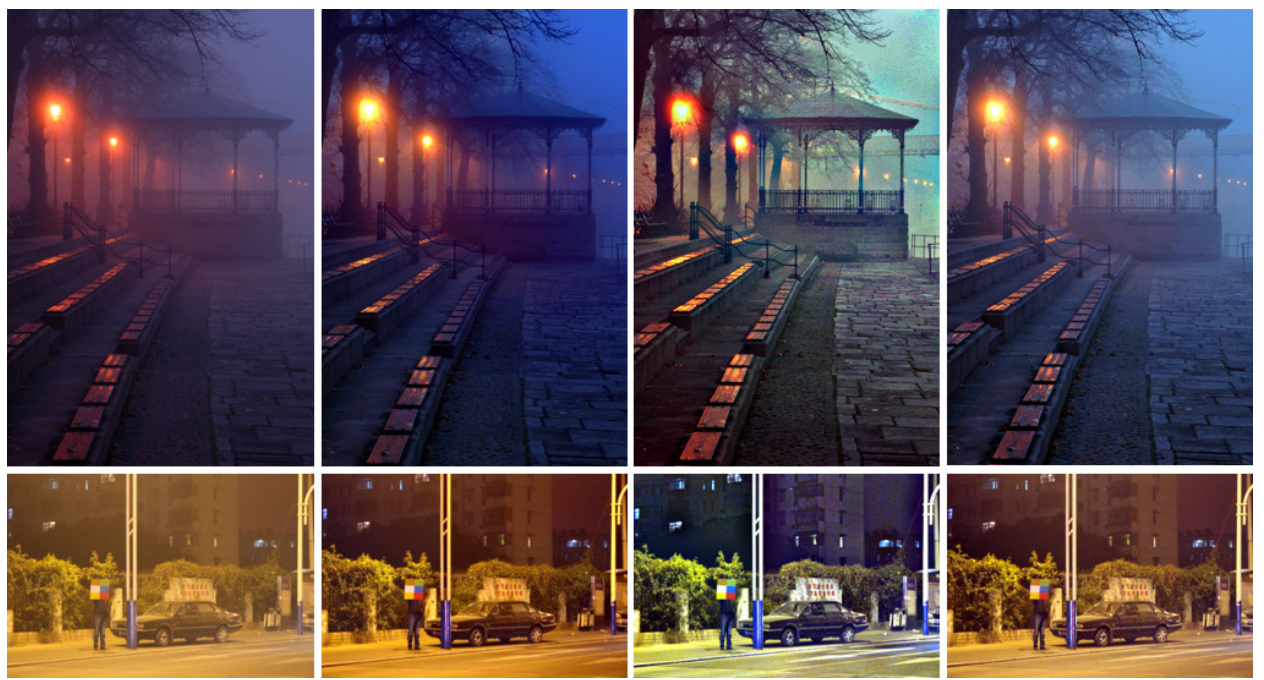

Figure 7: Night-time dehazing results. Left to Right, Input image, Haze-line [0], Li's method [四], Proposed method.

dehazing. In addition, $\bar{r}$ values in Table 1 show that the contrast of our haze-free images was improved.

As shown in the first row of Fig.6, the MSCNN and Color-line methods lost the details of the wood parts. The haze-line method was responsible for the halo effect at the edge. In the second row, the MSCNN and color-line method created an unnatural dark area as highlighted by the yellow rectangle. The third and fourth rows show the results of ny 12 and Urbino images, which had very bright regions near the sky. In color-line and haze-line methods, saturation occurred in these regions, as highlighted by the red rectangles. On the other hand, in the proposed method, no saturation problems occurred. Our algorithm preserved details of the image and produces better quality haze-free images. Fig.7 shows experimental results for the nighttime haze images. The existing algorithms were developed either for daytime or nighttime images. In the first row of Fig.7, Li's method [四] produced a good haze-free image, but failed to reflect the mood of the original night image. Additionally, artifacts occurred around street lamps due to the inaccurate global estimation. In the second row, the haze-line method produced several artifacts around the pillars. Since the haze-line method is originally the daytime algorithm, the haze removal results for nighttime were not good. Although the proposed method completely outperformed the existing night-time algorithms, experimental results show that our method reduced several artifacts and noise and produced better results than day-time algorithms. Please note that our method is applicable to both daytime and nighttime images because it uses the pixel-wise $A$.

\section{Conclusion}

This paper proposed a robust dehazing algorithm. We divided the image into two components, namely, illumination and reflectance, according to the retinex theory and obtained the pixel-wise atmospheric light from the illumination. In addition, the haze-relevant features, which were highly correlated with haze, were combined to estimate the initial transmission 
accurately. These processes were employed in the convex optimization framework to effectively deal with saturation and over-enhancement of the dark and bright areas of dehazing. The experimental results showed that the proposed algorithm was able to produce better qualitative and quantitative results. Future work should focus on finding a solution to the colour restoration problem and on reducing computational complexity.

\section{References}

[1] Codruta Ancuti, Cosmin Ancuti, Chris Hermans, and Philippe Bekaert. A fast semiinverse approach to detect and remove the haze from a single image. Computer VisionACCV 2010, pages 501-514, 2011.

[2] Codruta Orniana Ancuti and Cosmin Ancuti. Single image dehazing by multi-scale fusion. IEEE Transactions on Image Processing, 22(8):3271-3282, 2013.

[3] Cosmin Ancuti, Codruta O Ancuti, Christophe De Vleeschouwer, and Alan C Bovik. Night-time dehazing by fusion. In Image Processing (ICIP), 2016 IEEE International Conference on, pages 2256-2260. IEEE, 2016.

[4] D. Berman, T. Treibitz, and S. Avidan. Non-local image dehazing. In IEEE Conference on Computer Vision and Pattern Recognition (CVPR), 2016.

[5] Bolun Cai, Xiangmin Xu, Kui Jia, Chunmei Qing, and Dacheng Tao. Dehazenet: An end-to-end system for single image haze removal. IEEE Transactions on Image Processing, 25(11):5187-5198, 2016.

[6] Lark Kwon Choi, Jaehee You, and Alan Conrad Bovik. Referenceless prediction of perceptual fog density and perceptual image defogging. IEEE Transactions on Image Processing, 24(11):3888-3901, 2015.

[7] Faming Fang, Fang Li, Xiaomei Yang, Chaomin Shen, and Guixu Zhang. Single image dehazing and denoising with variational method. In Image Analysis and Signal Processing (IASP), 2010 International Conference on, pages 219-222. IEEE, 2010.

[8] Raanan Fattal. Single image dehazing. ACM transactions on graphics (TOG), 27(3): $72,2008$.

[9] Raanan Fattal. Dehazing using color-lines. volume 34, New York, NY, USA, 2014. ACM.

[10] Adrian Galdran, Javier Vazquez-Corral, David Pardo, and Marcelo Bertalmío. A variational framework for single image dehazing. In European Conference on Computer Vision, pages 259-270. Springer, 2014.

[11] Yuanyuan Gao, Hai-Miao Hu, Shuhang Wang, and Bo Li. A fast image dehazing algorithm based on negative correction. Signal Processing, 103:380-398, 2014.

[12] Tom Goldstein and Stanley Osher. The split bregman method for 11-regularized problems. SIAM journal on imaging sciences, 2(2):323-343, 2009. 
[13] Nicolas Hautiere, Jean-Philippe Tarel, Didier Aubert, and Eric Dumont. Blind contrast enhancement assessment by gradient ratioing at visible edges. Image Analysis \& Stereology, 27(2):87-95, 2011.

[14] Jiaxi He, Cishen Zhang, Ran Yang, and Kai Zhu. Convex optimization for fast image dehazing. In Image Processing (ICIP), 2016 IEEE International Conference on, pages 2246-2250. IEEE, 2016.

[15] Kaiming He, Jian Sun, and Xiaoou Tang. Single image haze removal using dark channel prior. CVPR, 2009.

[16] Jin-Hwan Kim, Jae-Young Sim, and Chang-Su Kim. Single image dehazing based on contrast enhancement. In Acoustics, Speech and Signal Processing (ICASSP), 2011 IEEE International Conference on, pages 1273-1276. IEEE, 2011.

[17] Jin-Hwan Kim, Won-Dong Jang, Jae-Young Sim, and Chang-Su Kim. Optimized contrast enhancement for real-time image and video dehazing. Journal of Visual Communication and Image Representation, 24(3):410-425, 2013.

[18] Johannes Kopf, Boris Neubert, Billy Chen, Michael Cohen, Daniel Cohen-Or, Oliver Deussen, Matt Uyttendaele, and Dani Lischinski. Deep photo: Model-based photograph enhancement and viewing. In ACM transactions on graphics (TOG), volume 27, page 116. ACM, 2008.

[19] Harald Koschmieder. Theorie der horizontalen sichtweite: kontrast und sichtweite. Keim \& Nemnich, 1925.

[20] Yu Li, Robby T Tan, and Michael S Brown. Nighttime haze removal with glow and multiple light colors. In Proceedings of the IEEE International Conference on Computer Vision, pages 226-234, 2015.

[21] Jiao Long, Zhenwei Shi, and Wei Tang. Fast haze removal for a single remote sensing image using dark channel prior. In Computer Vision in Remote Sensing (CVRS), 2012 International Conference on, pages 132-135. IEEE, 2012.

[22] Gaofeng Meng, Ying Wang, Jiangyong Duan, Shiming Xiang, and Chunhong Pan. Efficient image dehazing with boundary constraint and contextual regularization. In Proceedings of the IEEE international conference on computer vision, pages 617-624, 2013.

[23] Srinivasa G. Narasimhan and Shree K. Nayar. Contrast restoration of weather degraded images. IEEE transactions on pattern analysis and machine intelligence, 25(6):713724, 2003.

[24] Ko Nishino, Louis Kratz, and Stephen Lombardi. Bayesian defogging. International journal of computer vision, 98(3):263-278, 2012.

[25] Soo-Chang Pei and Tzu-Yen Lee. Nighttime haze removal using color transfer preprocessing and dark channel prior. In Image Processing (ICIP), 2012 19th IEEE International Conference on, pages 957-960. IEEE, 2012. 
[26] Wenqi Ren, Si Liu, Hua Zhang, Jinshan Pan, Xiaochun Cao, and Ming-Hsuan Yang. Single image dehazing via multi-scale convolutional neural networks. In European Conference on Computer Vision, pages 154-169. Springer, 2016.

[27] Lex Schaul, Clément Fredembach, and Sabine Süsstrunk. Color image dehazing using the near-infrared. In Image Processing (ICIP), 2009 16th IEEE International Conference on, pages 1629-1632. IEEE, 2009.

[28] Chih-Tsung Shen and Wen-Liang Hwang. Color image enhancement using retinex with robust envelope. In Image Processing (ICIP), 2009 16th IEEE International Conference on, pages 3141-3144. IEEE, 2009.

[29] Matan Sulami, Itamar Geltzer, Raanan Fattal, and Michael Werman. Automatic recovery of the atmospheric light in hazy images. In IEEE International Conference on Computational Photography (ICCP), 2014.

[30] Robby T Tan. Visibility in bad weather from a single image. In Computer Vision and Pattern Recognition, 2008. CVPR 2008. IEEE Conference on, pages 1-8. IEEE, 2008.

[31] Ketan Tang, Jianchao Yang, and Jue Wang. Investigating haze-relevant features in a learning framework for image dehazing. In Proceedings of the IEEE Conference on Computer Vision and Pattern Recognition, pages 2995-3000, 2014.

[32] Jean-Philippe Tarel and Nicolas Hautiere. Fast visibility restoration from a single color or gray level image. In Computer Vision, 2009 IEEE 12th International Conference on, pages 2201-2208. IEEE, 2009.

[33] Chunxia Xiao and Jiajia Gan. Fast image dehazing using guided joint bilateral filter. The Visual Computer, 28(6-8):713-721, 2012.

[34] Li Xu, Qiong Yan, Yang Xia, and Jiaya Jia. Structure extraction from texture via relative total variation. ACM Transactions on Graphics (TOG), 31(6):139, 2012.

[35] Chia-Hung Yeh, Li-Wei Kang, Ming-Sui Lee, and Cheng-Yang Lin. Haze effect removal from image via haze density estimation in optical model. Optics express, 21 (22):27127-27141, 2013. 